\title{
Outcomes of pediatric laparoscopic fundoplication: A critical review of the literature
}

\author{
Kathryn Martin MD, Catherine Deshaies MD CM, Sherif Emil MD CM
}

\begin{abstract}
KMartin, C Deshaies, S Emil. Outcomes of pediatric laparoscopic fundoplication: A critical review of the literature. Can J Gastroenterol Hepatol 2014;28(2):97-102.
\end{abstract}

BACKGROUND/OBJECTIVE: Laparoscopic fundoplication for gastroesophageal reflux disease (GERD) is one of the most common procedures performed in children. A critical literature review was performed to evaluate the level and quality of evidence supporting the efficacy of this procedure.

METHODS: Systematic reviews of the EMBASE, PubMed and CENTRAL databases were conducted to retrieve all articles published over a 15-year period (1996 to 2010) reporting medium- to long-term outcomes (minimum six months follow-up) of laparoscopic fundoplication for the treatment of pediatric GERD. Articles were critically appraised using the Newcastle-Ottawa quality assessment scale and the Cochrane risk of bias assessment tool. Extracted outcomes included GERD recurrence, need for reoperation, postoperative morbidity and mortality.

RESULTS: A total of 5302 articles were retrieved. Thirty-six studies met inclusion and exclusion criteria, including five prospective (level 2b), four retrospective comparative (level 3b) and 27 case series (level 4). No studies compared laparoscopic fundoplication with medical treatment. Thirty-six per cent of studies did not describe the symptoms used to suspect GERD; $11 \%$ did not disclose the diagnostic modalities used; and $41 \%$ did not report the findings of diagnostic modalities. Only $17 \%$ of studies provided a definition of recurrence, and only $14 \%$ attempted to control for confounding variables. The follow-up intervals were inconsistently reported, ranging between two months and nine years. Significant heterogeneity among studies limited the ability to pool outcomes. Mean $( \pm S D)$ recurrence rates varied between $0 \%$ and $48 \pm 19.6 \%$ of patients. Reoperation was required in $0.69 \pm 0.95 \%$ to $17.7 \pm 8.4 \%$ of patients. Mortality ranged between $0 \%$ and $24 \pm 16.7 \%$.

CONCLUSION: The level and quality of the evidence supporting laparoscopic fundoplication are extremely poor. Higher-quality data are required before the procedure can be considered to be an effective intervention in the treatment of pediatric GERD.

Key Words: Fundoplication; Gastroesophageal reflux disease; Laparoscopic; Pediatric

\section{Les résultats de la fundoplication laparoscopique en pédiatrie : une analyse bibliographique critique}

HISTORIQUE ET OBJECTIF : La fundoplication laparoscopique pour traiter le reflux gastro-œsophagien pathologique (RGOP) est l'une des interventions les plus effectuées chez les enfants. Les chercheurs ont procédé à une analyse bibliographique critique pour évaluer la qualité et la catégorie des preuves en corroborant l'efficacité.

MÉTHODOLOGIE : Les chercheurs ont procédé à l'analyse systématique des bases de données EMBASE, PubMedet CENTRAL pour en extraire tous les articles publiés sur une période de 15 ans (1996 à 2010) rendant compte des résultats à moyen et long terme (minimum de six mois de suivi) de la fundoplication laparoscopique pour traiter le RGOP en pédiatrie. Ils ont effectué l'évaluation critique des articles au moyen de l'échelle d'évaluation de la qualité de Newcastle-Ottawa et de l'outil d'évaluation du risque de biais de Cochrane. Les résultats obtenus incluaient la récurrence du RGOP, le besoin de réopérer, la morbidité postopératoire et la mortalité.

RÉSULTATS : Au total, les chercheurs ont extrait 5302 articles. Trente-six études respectaient les critères d'inclusion et d'exclusion, soit cinq études prospectives (catégorie $2 \mathrm{~b}$ ), quatre études comparatives prospectives (catégorie $3 b$ ) et 27 séries de cas (catégorie 4). Aucune étude ne comparait la fundoplication laparoscopique avec le traitement médical. Trente-six pour cent des études ne décrivaient pas les symptômes retenus pour présumer un RGOP, 11 \% ne révélaient pas les modalités diagnostiques utilisées et $41 \%$ ne précisaient pas les résultats des modalités diagnostiques. Seulement $17 \%$ des études incluaient une définition de la récurrence et seulement $14 \%$ cherchaient à contrôler les variables confusionnelles. Les intervalles de suivi, qui n'étaient pas transmis de façon uniforme, variaient entre deux mois et neuf ans. En raison de l'hétérogénéité importante des études, la possibilité de regrouper les résultats était limitée. Le taux de récurrence moyen ( \pm ÉT) variait entre $0 \%$ et $48 \pm 19,6 \%$ des patients.

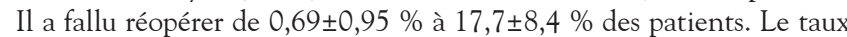
de mortalité se situait entre $0 \%$ et $24 \pm 16,7 \%$.

CONCLUSION : La qualité et la catégorie des preuves en appui à la fundoplication laparoscopique sont extrêmement faibles. Il faudra des données de meilleure qualité avant que l'intervention puisse être considérée comme efficace pour traiter le RGOP en pédiatrie.

Published reviews of LF have simply analyzed the data from individual studies without assessment of their quality (5-7). Position papers and treatment guidelines have been formulated by national and international surgical organizations based on the same data, typically without comment on the level of the evidence $(6,8)$. To fill this gap, we conducted a critical review of all articles published from 1996 to 2010 that reported the medium- to long-term outcomes of LF. Our primary goal was to assess the quality of the literature. Our secondary goal was to evaluate reported outcomes.

\section{METHODS}

Systematic searches of the EMBASE, PubMed and CENTRAL databases from 1996 to 2010 were conducted in conjunction with States.

Division of Pediatric General and Thoracic Surgery, The Montreal Children's Hospital; McGill University Health Centre, Montreal, Quebec

Correspondence: Dr Sherif Emil, Division of Pediatric General and Thoracic Surgery, Montreal Children's Hospital, 2300 Tupper Street, Room C-818,

Montreal, Quebec H3H 1P3. Telephone 514-412-4497, fax 514-412-4289, e-mail sherif.emil@mcgill.ca

Received for publication July 16, 2013. Accepted September 19, 2013 


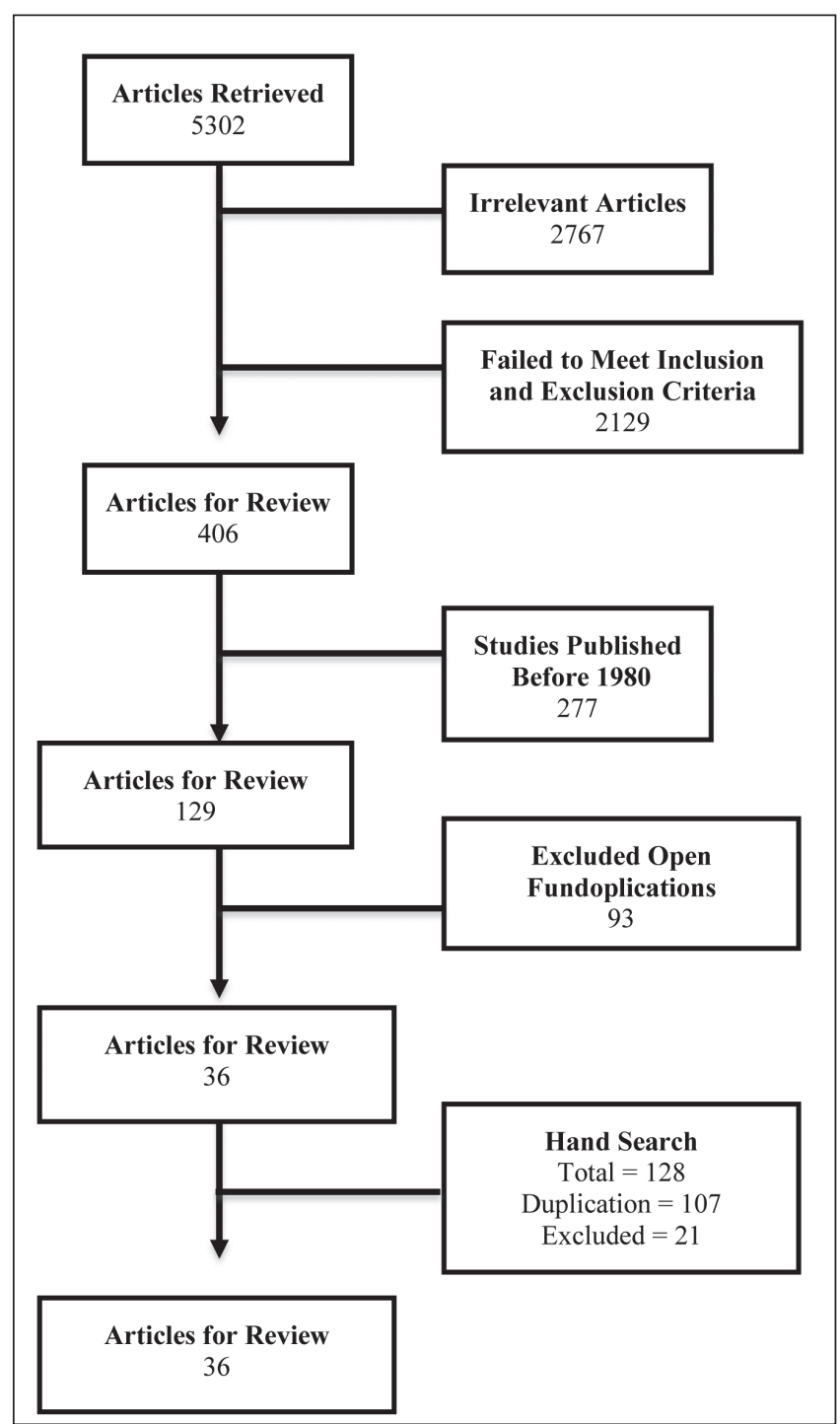

Figure 1) Article selection

librarians at the Montreal Children's Hospital, McGill University Health Centre (Montreal, Quebec). All studies reporting the mediumto long-term outcomes of LF in the treatment of pediatric GERD were retrieved. No language limitations were placed. Separate search strategies were formulated for each database using database-specific subject terms, syntax and free-text forms. Queries included a combination of exploded and nonexploded subject headings including the following: "gastroesophageal reflux disease", "GERD”, "fundoplication", "antireflux surgery" and "pediatrics". Review articles, case series involving $<20$ patients, editorials and letters were excluded. Articles reporting $<50 \%$ follow-up at six months were also excluded.

A single investigator reviewed all retrieved citations' titles and abstracts to eliminate those that clearly did not meet inclusion and exclusion criteria. Two independent reviewers reviewed the remaining articles in their entirety for final selection based on the standardized eligibility criteria. Any disagreements in the selection process were resolved through discussion between reviewers. The references of the selected articles were manually searched to identify any additional relevant articles.

The articles were reviewed in detail for quality assessment and data extraction. Data were then pooled and discrepancies dealt with through re-review until consensus was reached. Article quality was determined by study design, a standardized quality assessment form based on the Newcastle-Ottawa quality assessment scale and the
Cochrane risk-of-bias assessment tool. Data were extracted using a standardized data extraction form. Extracted data included baseline demographics, diagnostic criteria, study intervention, follow-up details and outcomes, including postoperative mortality, GERD recurrence and need for reoperation.

Quality assessments are reported qualitatively with special attention devoted to study design, confounders, biases and length of followup reporting. Outcomes are reported as proportions with 95\% Cls. Pooled estimates are presented where appropriate, based on $\chi^{2}$ testing for heterogeneity.

\section{RESULTS}

The literature search retrieved a total of 5302 articles, 36 of which met inclusion and exclusion criteria (Figure 1). A summary of the selected articles is presented in Table 1.

\section{Quality assessment}

The retrieved studies all constituted low-level evidence: five prospective comparative studies (level 2b) (9-13), four retrospective comparative studies (level 3b) (14-17) and 27 case series (level 4) (18-44). Three of the prospective studies compared LF in neurologically impaired versus neurologically intact patients. There were no randomized controlled trials of LF versus medical therapy. All nonrandomized comparative studies compared LF with open fundoplication or minor technical modifications in the laparoscopic technique; none compared LF with medical therapy. There were three before-and-after studies $(11-13)$. However, none of these studies clearly described the treatments, if any, that patients received before LF. Thus, the preoperative disease status was largely unknown, making the outcomes difficult to interpret (11-13).

Table 2 outlines the quality features of the selected articles. Only five studies attempted to control for confounders in their analysis. The factors controlled for varied among studies and included age, sex, comorbidities, respiratory status and surgical technique. The percentage of neurological impairment was reported in $78 \%$ of studies; however, the majority did not control for this factor in their analysis. Similarly, the rates of esophageal atresia were reported in $22 \%$ of studies, none of which controlled for its presence during analysis. No studies controlled for the confounding effects of the learning curve associated with LF.

Bias was present throughout, with only two studies clearly attempting to reduce bias $(10,33)$. The first was a prospective study involving a cohort of institutionalized patients (10). To reduce detection bias, this study used universal $\mathrm{pH}$ testing at 12 months postoperatively to detect recurrence and had no patients lost to follow-up. However, the authors did not describe how patients were assigned to LF, raising the possibility of a significant selection bias (10). The second study (33) was a case series that used clear selection criteria for $\mathrm{LF}$, routine $\mathrm{pH}$ testing at 12 months to detect recurrence and had no loss to follow-up.

The remaining studies were prone to selection, detection, reporting and attrition bias. With regard to selection bias, the studies failed to adequately describe why patients were selected for LF over other surgical or medical treatments. They also failed to describe the background population from which the reported samples were derived. Detection bias was prevalent due to the lack of a definition of recurrence, which was only present in $17 \%$ of studies, as well as the reliance on patientand caregiver-reported symptoms to trigger further investigations. Only two studies used a standardized follow-up interview with blinded assessors $(12,13)$. Reporting bias was prevalent because studies failed to indicate which outcomes were being investigated a priori. Furthermore, studies failed to report the absence of many common complications, making it unclear whether these did not occur, were not investigated or were omitted. Finally, attrition bias was a common problem because patients were lost to follow-up over time, particularly in studies reporting extended follow-up periods. All studies failed to indicate the features of participants lost to follow-up compared with those remaining in the sample. Follow-up protocols were rarely reported. 
TABLE 1

Summary of articles

\begin{tabular}{|c|c|c|c|c|}
\hline Author (reference), year & $\mathrm{n}$ & Study design & Population & Fundoplication technique \\
\hline Esposito et al (9), 2001 & 36 & Prospective cohort & $<1$ year of age & Nissen; Toupet \\
\hline Capito et al (11), 2008 & 127 & Prospective cohort & Mixed & Nissen-Rossetti \\
\hline Engelmann et al (13), 2010 & 26 & Prospective cohort & Mixed & Thal \\
\hline Somme et al (14), 2002 & 53 & Retrospective cohort & $<1$ year of age & Nissen \\
\hline Diaz et al (15), 2005 & 456 & Retrospective cohort & Mixed & Nissen \\
\hline Curtis et al (17), 2010 & 384 & Retrospective cohort & Mixed & Nissen \\
\hline Longis et al (18), 1996 & 30 & Case series & Mixed & Nissen-Rossetti; Toupet \\
\hline Thompson et al (19), 1996 & 25 & Case series & $<1$ year of age & Nissen \\
\hline Rothenberg et al (20), 1997 & 56 & Case series & Respiratory disease & Nissen; Nissen-Rossetti \\
\hline Tovar et al (21), 1998 & 27 & Case series & Mixed & Nissen \\
\hline Hopkins and Stringel (22), 1999 & 25 & Case series & Mixed & Nissen \\
\hline Allal et al (27), 2001 & 142 & Case series & Mixed & Nissen; Toupet \\
\hline Mattioli et al (28), 2002 & 70 & Case series & Mixed & Nissen-Rossetti \\
\hline Van der Zee et al (29), 2002 & 149 & Case series & Mixed & Nissen; Thal \\
\hline Pimpalwar et al (30), 2002 & 54 & Case series & Neurologically impaired & Nissen \\
\hline Mattioli et al (31), 2002 & 288 & Case series & Mixed & Nissen; Toupet; Thal \\
\hline Esposito et al (32), 2003 & 80 & Case series & Neurologically impaired & Nissen; Thal \\
\hline Mattioli et al (33), 2004 & 48 & Case series & Neurologically normal & Nissen-Rossetti \\
\hline Kwiecien et al (34), 2004 & 132 & Case series & Mixed & Toupet \\
\hline Lima et al (35), 2004 & 47 & Case series & Neurologically impaired & Nissen; Toupet; Dor \\
\hline Kawahara et al (36), 2004 & 56 & Case series & Neurologically impaired & Nissen; Thal \\
\hline
\end{tabular}

\section{Outcomes}

Mortality was reported by $58 \%$ of the studies. All mortalities were attributed to progression of the patients' underlying cardiac, neurological or respiratory disorders. Figure 2 illustrates the reported mortality rates with $95 \%$ Cls. The mean $( \pm$ SD) pooled mortality rate in neurologically impaired children was found to be $17.9 \pm 4.9 \%\left(\chi^{2}\right.$ for heterogeneity $\mathrm{P}=0.549$ ).

Recurrence rates following LF were reported by $83 \%$ of studies. However, only six studies used an explicit definition of recurrence. The reported recurrence rates ranged between $0 \%$ and $48 \pm 19.6 \%$ (Figure 3). Significant heterogeneity existed among the studies, preventing pooling of data $\left(\chi^{2}\right.$ for heterogeneity $\left.\mathrm{P}<0.001\right)$.

The need for reoperation following LF was reported in $50 \%$ of the studies. A consistent definition of indications for reoperation was absent. Some studies reported the percentage of re-do fundoplications due to recurrent GERD, while others included reoperation for wrap stenosis, pyloroplasty and recurrent hiatal hernia. The rate of reoperation was reported to be between $0.69 \pm 0.95 \%$ and $17.7 \pm 8.4 \%$ (Figure 4). Studies reporting neurologically impaired and neurologically normal populations were sufficiently homogenous to allow pooling ( $\chi^{2}$ for heterogeneity $\mathrm{P}=0.16$ and $\mathrm{P}=0.528$, respectively). The pooled estimate for reoperation in neurologically impaired patients was $15.4 \pm 4.2 \%$. The pooled estimate for reoperation in neurologically
TABLE 2

Summary of quality features

\begin{tabular}{lc}
\hline Quality feature & $\begin{array}{c}\text { Percentage } \\
\text { of studies }\end{array}$ \\
\hline Description of \% of population with neurological impairment & 78 \\
Description of \% of population with history of esophageal atresia & 22 \\
Description of patient symptoms & 64 \\
Description of diagnostic tests & 89 \\
Results of diagnostic tests & 52 \\
Description of surgical techniques & 75 \\
Median and range of follow-up provided & 33 \\
Relevant outcomes reported (recurrence, reoperation or death) & 100 \\
Clear definition of recurrence given & 17 \\
Details regarding complications & 92 \\
Attempts to control for confounders & 14 \\
Attempts to reduce bias & 6 \\
\hline
\end{tabular}

normal patients was $7.0 \pm 3.3 \%$. The neurologically impaired group underwent significantly more reoperations than the neurologically normal group $\left(\chi^{2} \mathrm{P}=0.003\right)$. 


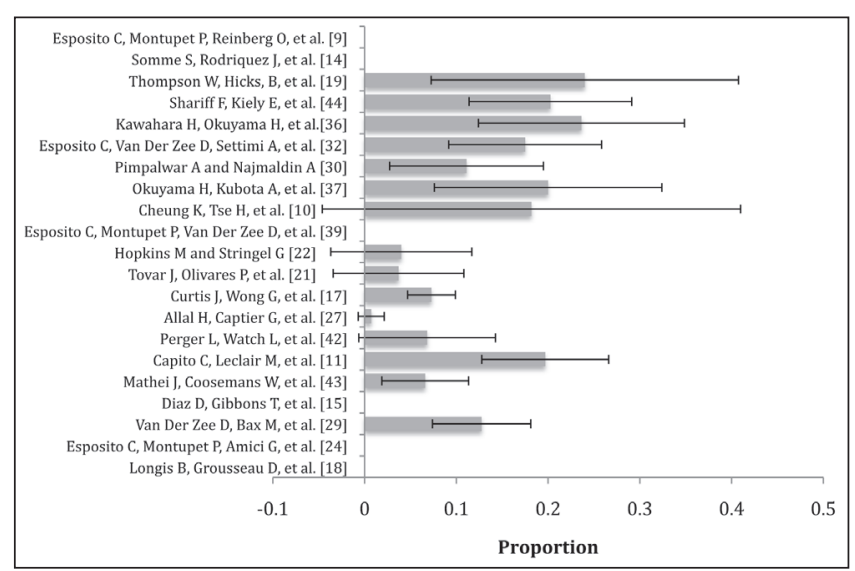

Figure 2) Mortality rates. Bars represent 95\% CIs

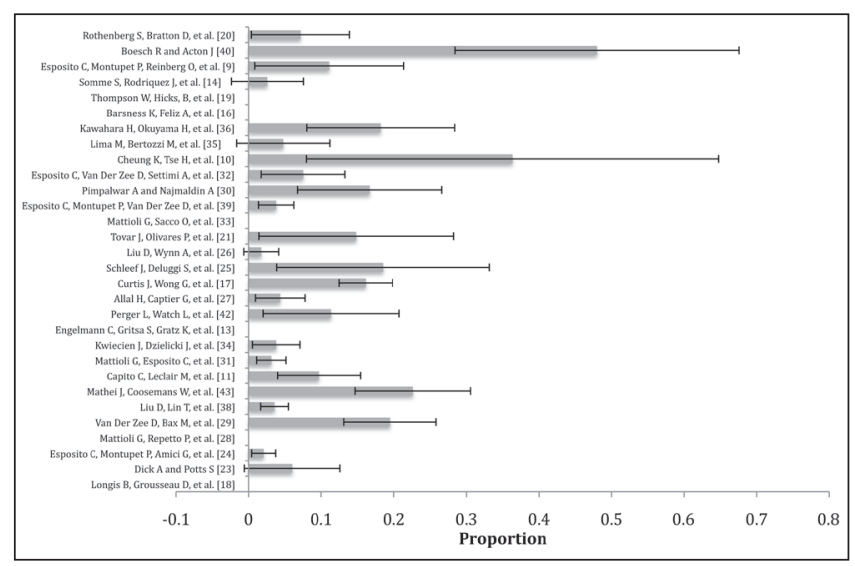

Figure 3) Recurrence rates. Bars represent 95\% CIs

\section{DISCUSSION}

LF is the current standard surgical treatment for refractory pediatric GERD. However, little is known about the long-term outcomes of this procedure and its true effectiveness. The available literature is of extremely poor quality according to evidence-based standards. Presently, there are no randomized trials or prospective cohort studies comparing LF with medical therapy. Thus, there is no conclusive evidence that surgery is superior to medical therapy (4).

In our review, $75 \%$ of studies were case series, which are known to favour the described intervention. They lead to false inferences up to $50 \%$ of the time (45). Multiple innovative treatments, once believed to be effective based on case series, were subsequently found to be no better than standard treatments when rigorously studied (45). Although nine of the included studies were comparative, none were randomized and three used historical controls. The use of historical controls is often confounded by changes in management, separate from the intervention in question, leading to false inferences in $40 \%$ to $60 \%$ of cases (45).

Studies frequently failed to adequately describe their study populations, diagnostic criteria, follow-up protocols and outcome measures. The majority of studies indicated the proportion of the study population that was neurologically impaired. However, most failed to analyze this population separately, despite its association with worse outcomes (46). We found a statistically significant difference in reoperation rates between these two groups in our study. Similar concerns exist over studies including patients with esophageal atresia (3). Mixed populations were reported by $57 \%$ of reviewed studies. The failure to report and control for underlying comorbidities makes it difficult to apply results to a given patient population (3).

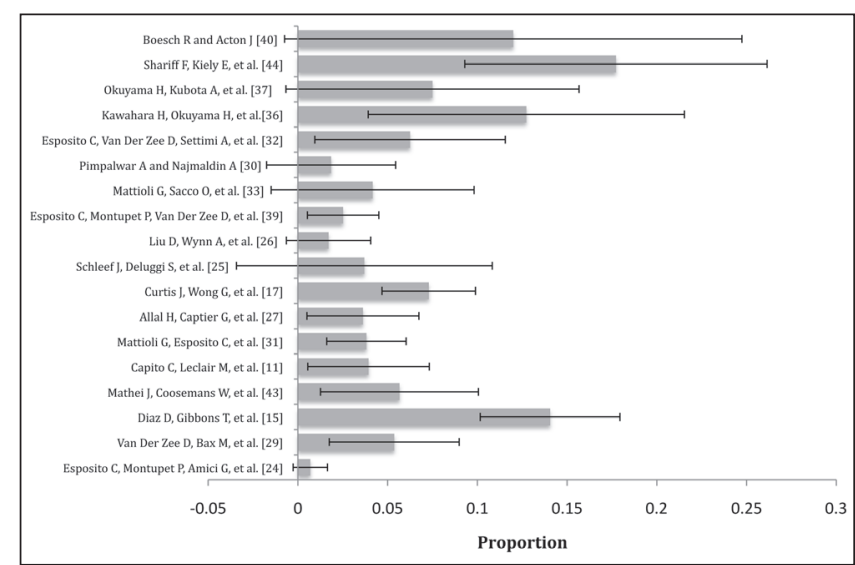

Figure 4) Reoperation rates. Bars represent 95\% CIs

There was also a consistent failure to explicitly outline the diagnostic criteria of GERD, the selection criteria for intervention and the outcome measures used. The lack of criteria for diagnosis and treatment likely relates to the lack of a standard definition of GERD in the pediatric population. An international panel of experts created guidelines in 2009 (1); however, most studies were published before 2009. Furthermore, these guidelines have yet to be universally adopted; therefore, recent studies fail to apply them $(1,47)$. Comparing studies was further hindered by the lack of clear, a priori outcomes and standardized follow-up protocols. Many have called for the use of standardized outcome measures when reporting the efficacy of GERD treatments (3,47-49). However, as noted by Gold et al (49), validated outcome measures do not currently exist. Finally, patient- and parentreported outcomes were heavily used in this body of literature, increasing the risk of a placebo effect, especially given the subjective nature of many of the reported outcomes. This is compounded by a potential second placebo response due to the natural history of GERD in children, the efficacy of nonpharmacological methods and expectation bias (50). All of these factors likely overestimate the true effectiveness of LF.

The biases present in the analyzed studies further limit the conclusions that can be drawn from them. Selection bias was present because clear inclusion and exclusion criteria were lacking from the majority of the studies. Thus, surgeons were likely to include patients they believed would benefit most from LF, and exclude those considered to be at increased risk of complications. The lack of clear a priori followup protocols means that many complications were likely not detected, not reported or both. Significant attrition bias existed due to the failure to account for patients lost to follow-up. The proportion lost to follow-up, as well as the reasons for that loss, was not reported by any of the reviewed studies.

Recurrence rates varied widely. The largest study of open fundoplication, a multicentre retrospective series of 7467 cases (51), reported a recurrence rate of $7 \%$. However, this was probably a gross underestimation because the study was poorly designed to detect recurrence. Objective testing for GERD using esophagogastroduodenoscopy or $\mathrm{pH}$ probe was only performed in $54 \%$ and $26 \%$, respectively, and the results of this objective testing were not reported (51). The study presented no standard follow-up, no standard assessment of outcomes and no quality of life measures (51). The wide range of recurrences in the current review are likely due to variations in the definition of recurrence and generally poor follow-up data. Studies with very high recurrence rates may have erroneously implicated GERD as the reason for the patient's symptoms $(5,52)$. For example, a recurrence rate of $48 \pm 19.6 \%$ was found in a population with respiratory disease attributed to GERD (40). The link between respiratory disease and GERD is not firmly established, and several studies have shown no benefit to LF when performed for respiratory indications $(48,53,54)$. 
Reoperation was required in all 18 studies reporting this outcome. The indications for reoperation varied among studies and included recurrence, wrap failure, esophageal stenosis, recurrent hiatal hernia and pyloroplasty for postoperative delayed gastric emptying. The pooled estimate of reoperation rate in neurologically impaired patients was $15.4 \pm 4.2 \%$ versus $7.0 \pm 3.3 \%$ in neurologically normal patients. Neurologically impaired patients also had the highest mortality rates, along with patients undergoing fundoplication before their first birthday $(19,36)$. These differences support the notion that neurologically impaired patients experience worse outcomes after LF (55). LF should be considered a palliative procedure for many of these patients and the surgeon should openly share the outcome information to provide the family with realistic expectations.

Prospective studies comparing LF with medical therapies are needed. Although, a randomized controlled trial would be optimal, it is unlikely to occur due to the wide adoption of LF by surgeons and parents alike as a common treatment option for pediatric GERD. A well-designed multicentre prospective cohort study using matched controls is a more realistic option. Such a study should aim to provide long-term follow-up and provide subgroup analysis to determine the long-term effects of LF in different populations. In the meantime, adoption of a universal definition of GERD may allow more objective selection of patients for LF (47). The use of standard outcome measures, as suggested by Gold et al (49), can create uniformity in the literature, allowing meaningful comparisons among studies. The enthusiasm for LF in children should be tempered until higher-quality evidence is available to support its long-term efficacy.

\section{SUMMARY}

Pediatric LF is one of the most common operative procedures performed in children. Multiple case series and several reviews of pediatric LF have been published over the past 15 years. However, none of the reviews critically evaluated the quality of the literature from an evidence-based perspective. We performed a critical review of the literature on pediatric LF outcomes over a 15 -year period. Our results indicate that the level and quality of the evidence supporting LF are extremely poor. Higher-quality data are required before the procedure can be considered to be an effective intervention for the treatment of pediatric GERD.

\section{REFERENCES}

1. Vandenplas Y, Rudolph C, Di Lorenzo C, et al. Pediatric gastroesophageal reflux clinical practice guidelines: Joint recommendations of the North American Society for Pediatric Hastroenterology, Hepatology, and Nutrition (NASPGHAN) and the European Society for Pediatric Gastroenterology, Hepatology, and Nutrition (ESPGHAN). J Pediatr Gastroenterol Nutr 2009;49:498-547.

2. Hassall E. Step-up and step-down approaches to treatment of gastroesophageal reflux disease in children. Curr Gastroenterol Rep 2008; 10:324-31.

3. Hassall E. Outcomes of fundoplication: Causes for concern, newer options. Arch Dis Child 2005;90:1047-52.

4. Kumar Y, Sarvananthan R. GORD in children. Clin Evid 2008; $10: 310$.

5. Lobe T. The current role of laparoscopic surgery for gastroesophageal reflux disease in infants and children. Surg Endosc 2007;21:167-74.

6. Kane T, Brown M, Chen M, et al. Position paper on laparoscopic antireflux operations in infants and children for gastroesophageal reflux disease. J Pediatr Surg 2009;44:1034-40.

7. Rafay M, Siddiqui S, Abdulaal Y, et al. A meta-analysis of outcomes after open and laparoscopic Nissen's fundoplication for gastroesophageal reflux disease in children. Pediatr Surg Int 2011;27:359-66.

8. IPEG guidelines for the surgical treatment of pediatric gastroesophageal reflux disease (GERD). J Laparoendosc Adv Surg Tech 2008;18:x-xiii.

9. Esposito C, Montupet P, Reinberg O. Laparoscopic surgery for gastroesophageal reflux disease during the first year of life. J Pediatr Surg 2001;36:715-7.
10. Cheung KM, Tse HW, Tse PW, et al. Nissen fundoplication and gastrostomy in severely neurologically impaired children with gastroesophageal reflux. Hong Kong Med J 2006;12:282-8.

11. Capito C, Leclair MD, Piloquet $H$, et al. Long-term outcome of laparoscopic Nissen-Rossetti fundoplication for neurologically impaired and normal children. Surg Endosc 2008;22:875-80.

12. Engelmann C, Gritsa S, Ure BM. Impact of laparoscopic anterior 270 degrees fundoplication on the quality of life and symptoms profile of neurodevelopmentally delayed versus neurologically unimpaired children and their parents. Surg Endosc 2010;24:1287-95.

13. Engelmann C, Gritsa S, Gratz KF, et al. Laparoscopic anterior hemifundoplication improves key symptoms without impact on GE in children with and children without neurodevelopmental delays. J Pediatr Gastroenterol Nutr 2010;51:437-42.

14. Somme S, Rodriguez JA, Kirsch DG, et al. Laparoscopic versus open fundoplication in infants. Surg Endosc 2002;16:54-6.

15. Diaz DM, Gibbons TE, Heiss K, et al. Antireflux surgery outcomes in pediatric gastroesophageal reflux disease. Am J Gastroenterol 2005;100:1844-52.

16. Barsness KA, Feliz A, Potoka DA, et al. Laparoscopic versus open Nissen fundoplication in infants after neonatal laparotomy. JSLS 2007;11:461-5.

17. Curtis JL, Wong G, Gutierrez I, et al. Pledgeted mattress sutures reduce recurrent reflux after laparoscopic Nissen fundoplication. J Pediatr Surg 2010;45:1159-64.

18. Longis B, Grousseau D, Alain JL, et al. Laparoscopic fundoplication in children: Our first 30 cases. J Laparoendosc Surg Adv Surg Tech 1996;6:S21-S29.

19. Thompson WR, Hicks BA, Guzzetta PC. Laparoscopic Nissen fundoplication in the infant. J Laparoendosc Surg Adv Surg Tech 1996;6:S5-7.

20. Rothenberg SS, Bratton D, Larsen G, et al. Laparoscopic fundoplication to enhance pulmonary function in children with severe reactive airway disease and gastroesophageal reflux disease. Surg Endosc 1997;11:1088-90.

21. Tovar JA, Olivares P, Diaz M, et al. Functional results of laparoscopic fundoplication in children. J Pediatr Gastroenterol Nutr 1998;26:429-31.

22. Hopkins MA, Stringel G. Laparoscopic Nissen fundoplication in children: A single surgeon's experience. JSLS. 1999;3:261-6.

23. Dick AC, Potts SR. Laparoscopic fundoplication in children - an audit of fifty cases. Eur J Pediatr Surg 1999;9:286-8.

24. Esposito C, Montupet P, Amici G, et al. Complications of laparoscopic antireflux surgery in childhood. Surg Endosc 2000;14:622-4.

25. Schleef J, Deluggi S, Schaarschmidt K, et al. Multi-institutional experience in laparoscopic surgery for gastroesophageal reflux: A five-year experience with 30 children. Pediatr Endosurg Innov Tech 2000;4:265-70.

26. Liu DC, Wynn AR, Rodriguez JA, et al. Laparoscopic NissenRossetti fundoplication in children. Pediatr Endosurg Innov Tech 2001;5:19-22.

27. Allal H, Captier G, Lopez M, et al. Evaluation of 142 consecutive laparoscopic fundoplications in children: Effects of the learning curve and technical choice. J Pediatr Surg 2001;36:921-6.

28. Mattioli G, Repetto P, Leggio S, et al. Laparoscopic Nissen-Rossetti fundoplication in children. Semin Laparosc Surg 2002;9:153-62.

29. van der Zee DC, Bax KN, Ure BM, et al. Long-term results after laparoscopic Thal procedure in children. Semin Laparosc Surg 2002;9:168-71

30. Pimpalwar A, Najmaldin A. Results of laparoscopic antireflux procedures in neurologically impaired children. Semin Laparosc Surg 2002;9:190-6.

31. Mattioli G, Esposito C, Lima M, et al. Italian multicenter survey on laparoscopic treatment of gastro-esophageal reflux disease in children. Surg Endosc 2002;16:1666-8.

32. Esposito C, van der Zee DC, Settimi A, et al. Risks and benefits of surgical management of gastroesophageal reflux in neurologically impaired children. Surg Endosc 2003;17:708-10.

33. Mattioli G, Sacco O, Gentilino V, et al. Outcome of laparoscopic Nissen-Rossetti fundoplication in children with gastroesophageal reflux disease and supraesophageal symptoms. Surg Endosc 2004;18:463-5.

34. Kwiecien J, Dzielicki J, Korlacki W, et al. Long-term effects of laparoscopic antireflux surgery in children with gastroesophageal reflux disease. Pediatria Wspolczesna 2004;6:249-53. 
35. Lima M, Bertozzi M, Ruggeri G, et al. Laparoscopic antireflux surgery in neurologically impaired children. Pediatr Surg Int 2004;20:114-7.

36. Kawahara H, Okuyama H, Kubota A, et al. Can laparoscopic antireflux surgery improve the quality of life in children with neurologic and neuromuscular handicaps? J Pediatr Surg 2004;39:1761-4.

37. Okuyama H, Kubota A, Kawahara H, et al. The efficacy and longterm outcome of laparoscopic Nissen fundoplication in neurologically impaired children. Pediatr Endosurg Innov Tech 2004;8:5-11.

38. Liu DC, Lin T, Statter MB, et al. Laparoscopic Nissen fundoplication without division of short gastric vessels in children. J Pediatr Surg 2006;41:120-5.

39. Esposito C, Montupet P, van der Zee D, et al. Long-term outcome of laparoscopic Nissen, Toupet, and Thal antireflux procedures for neurologically normal children with gastroesophageal reflux disease. Surg Endosc 2006;20:855-8.

40. Boesch RP, Acton JD. Outcomes of fundoplication in children with cystic fibrosis. J Pediatr Surg 2007;42:1341-4.

41. Tannuri AC, Tannuri U, Mathias AL, et al. Gastroesophageal reflux disease in children: Efficacy of Nissen fundoplication in treating digestive and respiratory symptoms. Experience of a single center. Dis Esophagus 2008;21:746-50.

42. Perger L, Watch L, Weinsheimer R, et al. Laparoscopically supervised PEG at time of Nissen fundoplication: A safe option. J Laparoendosc Adv Surg Tech 2008;18:136-9.

43. Mathei J, Coosemans W, Nafteux P, et al. Laparoscopic Nissen fundoplication in infants and children: Analysis of 106 consecutive patients with special emphasis in neurologically impaired vs. neurologically normal patients. Surg Endosc 2008;22:1054-9.

44. Shariff F, Kiely E, Curry J, et al. Outcome after laparoscopic fundoplication in children under 1 year. J Laparoendosc Adv Surg Tech 2010;20:661-4.
45. Frader JE, Flanagan-Klygis E. Innovation and research in pediatric surgery. Semin Pediatr Surg 2001;10:198-203.

46. Pearl R, Robie D, Ein S, et al. Complications of gastroesophageal antireflux surgery in neurologically impaired versus neurologically normal children. J Pediatr Surg 1990;25:1169-73.

47. Sherman P, Hassall E, Fagundes-Neto U, et al. A global, evidencebased consensus on the definition of gastroesophageal reflux disease in the pediatric population. Am J Gastroenterol 2009;104:1278-95.

48. Tolia V, Vandenplas Y. Systematic review: The extra-oesophageal symptoms of gastro-oesophageal reflux disease in children. Aliment Pharmacol Ther 2009;29:258-72.

49. Gold B, Co J, Colletti R, et al. What outcome measures are needed to assess gastroesophageal reflux disease in chidlren? What study design is appropriate? What new knowledge is needed? J Pediatr Gastroenterol Nutr 2003;37:S72-75.

50. Orenstein S, Hassall E, Furmaga-Jablonska W, et al. Multicenter, double-blind, randomized, placebo-controlled trial assessing the efficacy and safety of proton pump inhibitor lansoprazole in infants with symptoms of gastroesophageal reflux disease. J Pediatr 2009;154:514-20.

51. Fonkalsrud EW, Ashcraft KW, Coran AG, et al. Surgical treatment of gastroesophageal reflux in children: A combined hospital study of 7467 patients. Pediatrics 1998;10:419-22.

52. Di Lorenzo C, Orenstein S. Fundoplication: Friend or foe? J Pediatr Gastroenterol Nutr 2002;34:117-24.

53. Thakkar K, Boatright R, Gilger M, et al. Gastroesophageal reflux and asthma in children: A systematic review. Pediatrics 2010;125:e925-e930.

54. Lee S, Shabatian H, Hsu J, et al. Hospital admissions for respiratory symptoms and failure to thrive before and after Nissen fundoplication. J Pediatr Surg 2008;43:59-65.

55. de Veer A, Bos J, Niezen-de Boer R, et al. Symptoms of gastroesophageal reflux disease in severely mentally retarded people: A systematic review. BMC Gastroenterol 2008;8:23. 


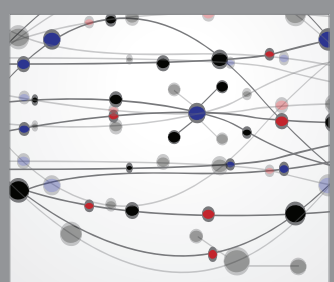

The Scientific World Journal
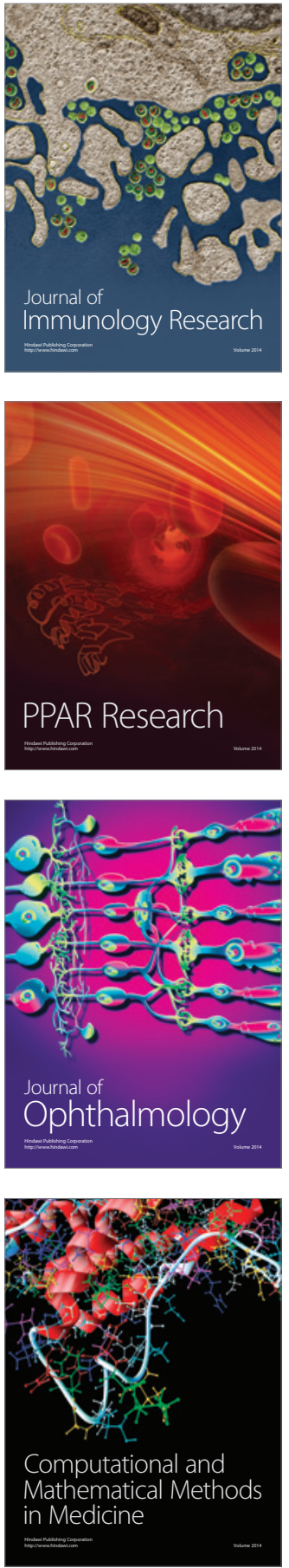

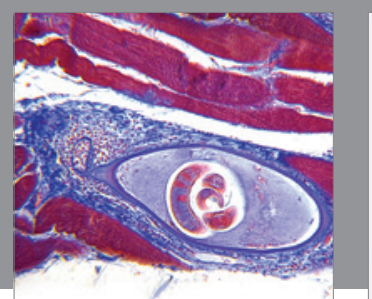

Gastroenterology Research and Practice

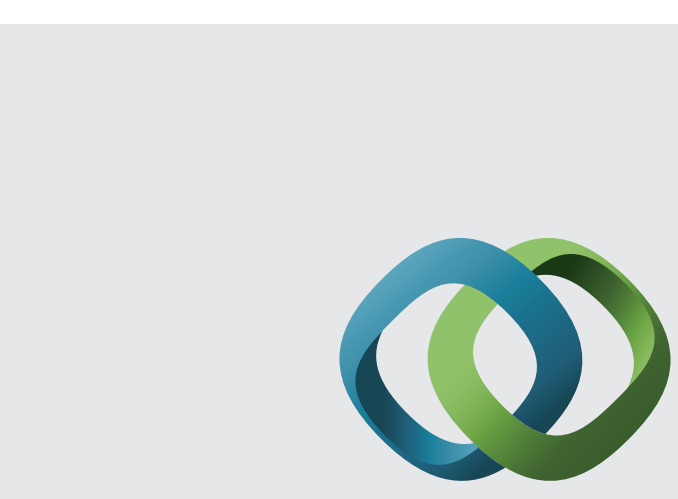

\section{Hindawi}

Submit your manuscripts at

http://www.hindawi.com
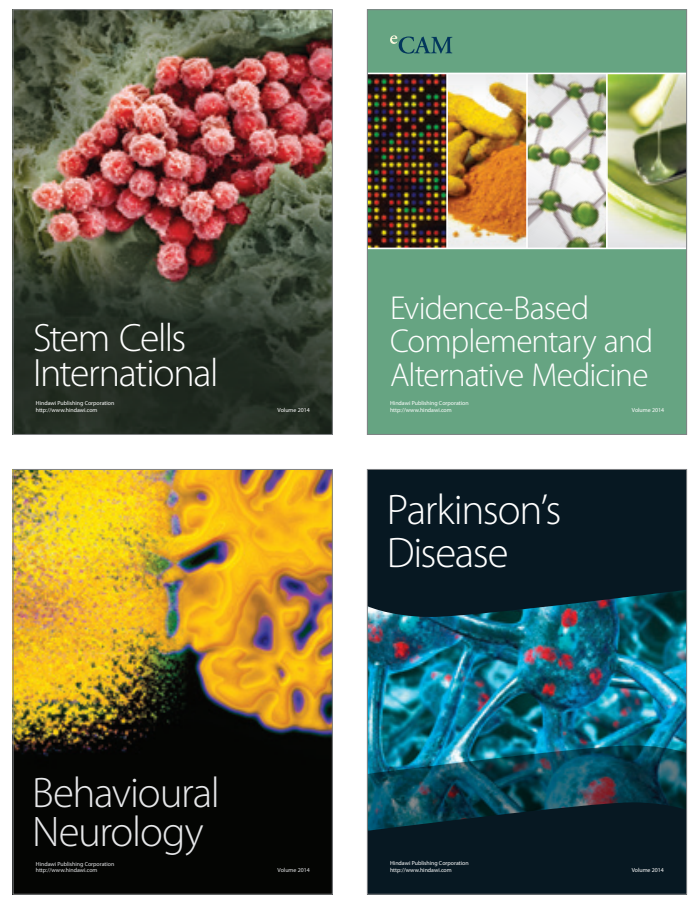
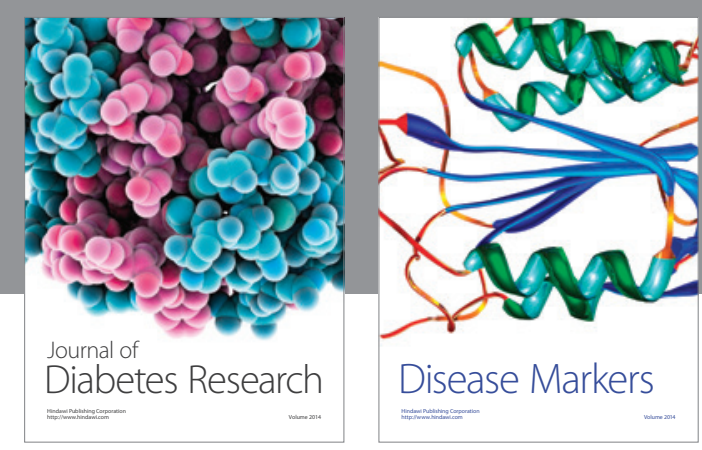

Disease Markers
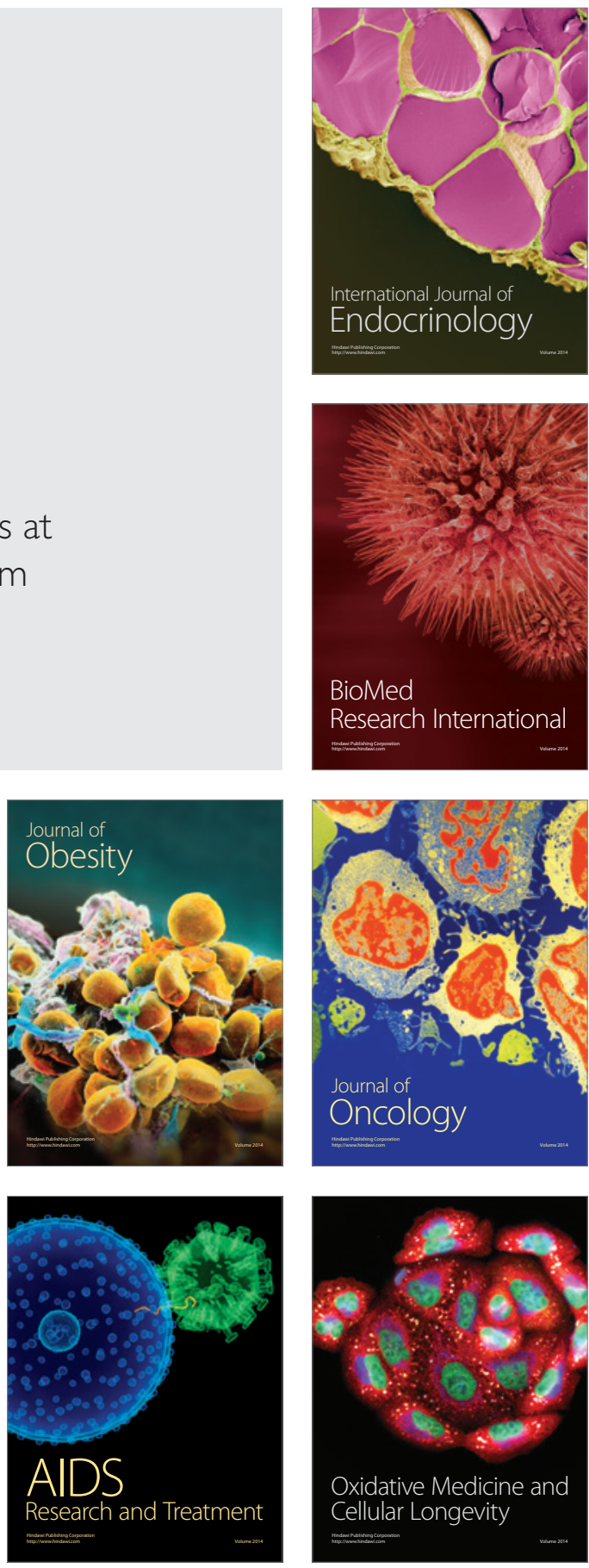